\title{
Macitentan for the treatment of idiopathic pulmonary fibrosis: the randomised controlled MUSIC trial
}

\author{
Ganesh Raghu1, Rachel Million-Rousseau², Adele Morganti, Loïc Perchenet², \\ Juergen Behr ${ }^{4}$ and the MUSIC Study Group ${ }^{5}$
}

Affiliations: ${ }^{1}$ Division of Pulmonary and Critical Care Medicine, University of Washington, Seattle, WA, USA. ${ }^{2}$ Dept of Clinical Science, Actelion Pharmaceuticals Ltd, Allschwil, and ${ }^{3}$ Dept of Biostatistics, Actelion Pharmaceuticals Ltd, Allschwil, Switzerland. “Dept of Internal Medicine V, Comprehensive Pneumology Centre, University of Munich, Munich, Germany. ${ }^{5} \mathrm{~A}$ full list of the MUSIC Study Group members and their affiliations can be found in the Acknowledgements section.

Correspondence: J. Behr, Dept of Internal Medicine V, Comprehensive Pneumology Centre, Member of the DZL, University of Munich, Marchioninistr 15, 81377 Munich, Germany.

E-mail: Juergen.behramed.uni-muenchen.de

ABSTRACT Idiopathic pulmonary fibrosis is a progressive, fatal disease. This prospective, randomised, double-blind, multicentre, parallel-group, placebo-controlled phase II trial (NCT00903331) investigated the efficacy and safety of the endothelin receptor antagonist macitentan in idiopathic pulmonary fibrosis.

Eligible subjects were adults with idiopathic pulmonary fibrosis of $<3$ years duration and a histological pattern of usual interstitial pneumonia on surgical lung biopsy. The primary objective was to demonstrate that macitentan (10 mg once daily) positively affected forced vital capacity versus placebo.

Using a centralised system, 178 subjects were randomised $(2: 1)$ to macitentan $(n=119)$ or placebo $(n=59)$. The median change from baseline up to month 12 in forced vital capacity was $-0.20 \mathrm{~L}$ in the macitentan arm and $-0.20 \mathrm{~L}$ in the placebo arm. Overall, no differences between treatments were observed in pulmonary function tests or time to disease worsening or death. Median exposures to macitentan and placebo were 14.5 months and 15.0 months, respectively. Alanine and/or aspartate aminotransferase elevations over three times upper limit of normal arose in 3.4\% of macitentan-treated subjects and $5.1 \%$ of placebo recipients.

In conclusion, the primary objective was not met. Long-term exposure to macitentan was well tolerated with a similar, low incidence of elevated hepatic aminotransferases in each treatment group.

@ERSpublications

Long-term exposure to macitentan was well tolerated in IPF in a trial that did not meet its primary end-point http://ow.ly/p0RDL

For editorial comments see page 1446

This article has supplementary material available from www.erj.ersjournals.com

Received: July 062012 | Accepted after revision: April 102013 | First published online: May 162013

Clinical trial: This study is registered at www.clinicaltrials.gov with identifier number NCT00903331.

Support statement: Funding for the Macitentan Use in an Idiopathic Pulmonary Fibrosis Clinical (MUSIC) trial was provided by Actelion Pharmaceuticals Ltd (Allschwil, Switzerland). Representatives of Actelion Pharmaceuticals Ltd participated in the study design and the collection, analysis and the interpretation of data. Medical writing assistance during the preparation of this manuscript was funded by Actelion Pharmaceuticals Ltd.

Conflict of interest: Disclosures can be found alongside the online version of this article at www.erj.ersjournals.com

Copyright @ERS 2013 


\section{Introduction}

Idiopathic pulmonary fibrosis (IPF) is a chronic fibrosing lung disease [1]. IPF occurs predominantly in older adult males and is characterised by radiological and/or histopathological patterns of usual interstitial pneumonia (UIP), progressive worsening of dyspnoea and lung function and a poor prognosis [1, 2]. A limited number of studies have suggested potential benefits from some pharmacological agents in IPF treatment [3-6].

While the pathogenesis of IPF is incompletely understood, the potent vasoconstrictor and growth factor endothelin-1 has been implicated in the fibrogenic processes underlying the disease [7]. However, two previously reported trials of the oral dual endothelin receptor antagonist (ERA) bosentan in subjects with IPF did not meet their primary end-points $[8,9]$ and a trial of the single ERA ambrisentan was terminated due to a lack of efficacy [10].

Macitentan, a novel dual ERA, has been shown to prevent the development of lung fibrosis in a rat model of bleomycin-induced pulmonary fibrosis [11]. To investigate the efficacy and safety of macitentan in subjects with IPF, the prospective, randomised, double-blind, multicentre, parallel-group, placebo-controlled, phase II, proof-of-concept Macitentan USe in an Idiopathic Pulmonary Fibrosis Clinical (MUSIC) trial (NCT00903331) was designed.

\section{Material and methods \\ Study subjects}

Subjects were eligible to participate in the trial if they were aged $\geqslant 18$ years with a proven diagnosis of IPF, according to the American Thoracic Society/European Respiratory Society statement [12], of $<3$ years duration, with surgical lung biopsy features of UIP.

During assessment for eligibility, prospective subjects were excluded prior to randomisation if they exhibited any of the following: interstitial lung disease due to conditions other than IPF; extensive honeycombing on baseline high-resolution computed tomography (as defined by the criteria used in the Bosentan Use in Interstitial Lung Disease (BUILD)-3 trial [9]); forced vital capacity (FVC) $<50 \%$ predicted or $<1.2 \mathrm{~L}$; diffusing capacity of the lung for carbon monoxide corrected for haemoglobin (DLCO) $<30 \%$ pred; residual volume $\geqslant 120 \%$ pred; or obstructive lung disease (defined as forced expiratory volume in $1 \mathrm{~s}$ $\left.\left(\mathrm{FEV}_{1}\right) / \mathrm{FVC}<0.70\right)$. A full list of exclusion criteria is available as online supplementary material.

In addition, subjects were ineligible for participation in the trial if, within 4 weeks of randomisation, they received any of the following treatments: ERAs; oral corticosteroids $\left(>20 \mathrm{mg} \cdot \mathrm{day}^{-1}\right.$ prednisone or equivalent); immunosuppressive or cytotoxic drugs (including cyclophosphamide and azathioprine); antifibrotic drugs (including pirfenidone, D-penicillamine, colchicine, tumour necrosis factor- $\alpha$ antagonists, imatinib, interferon- $\gamma$ ); $\mathrm{N}$-acetylcysteine ( $>600 \mathrm{mg} \cdot \mathrm{day}^{-1}$ prescribed chronically for IPF); or oral anticoagulants (where prescribed for IPF).

The MUSIC trial was conducted in academic and community hospitals in selected countries and in conformance with the principles of the Declaration of Helsinki. Approval from all relevant ethics committees and institutional review boards was received prior to study start. Written, informed consent was obtained from all subjects. The trial was registered at ClinicalTrials.gov (NCT00903331).

\section{Study design}

The MUSIC trial was a prospective, randomised, double-blind, multicentre, parallel-group, placebocontrolled, phase II study. The primary objective was to demonstrate that macitentan (10 mg once daily) positively affected FVC versus placebo. Secondary objectives were to evaluate the effects of macitentan on the time to IPF worsening or death and to evaluate the safety and tolerability of macitentan in a population of subjects with IPF.

Full details of the study assessments performed at each visit are available as online supplementary material. In brief, subjects were assessed at screening, randomisation and every 4 months thereafter until the end of the study.

Screening assessments included demographics, medical history, vital signs, concomitant medications, laboratory tests, pulmonary function tests, resting arterial blood gas, assessment of high-resolution computed tomography of the chest and histological analysis of surgical lung biopsy specimens.

At time of randomisation, subjects were assessed using the baseline dyspnoea index [13], vital signs, physical examination, concomitant medications, FEV1, FVC, corrected DLCO and laboratory tests. Assessments at 
4-monthly intervals until the end of treatment included FEV1, FVC, corrected DLCO, transition dyspnoea index (TDI), laboratory tests, concomitant medications and adverse events.

The end-of-study visit took place when the last randomised subject who did not discontinue treatment had completed 12 months of therapy. At this visit, FEV1, FVC, corrected DLCO, TDI, concomitant medications for IPF and disease-worsening events were assessed.

Measurements for the investigation of safety and tolerability were documented throughout the study with a final assessment at 28 days after study drug discontinuation. Subjects were followed until the end of the study even if they discontinued treatment.

The planned sample size for this trial was 156 subjects, with 104 subjects required to receive macitentan and 52 required to receive placebo. This sample size was considered necessary to detect with $80 \%$ power a placebo-corrected mean reduction in FVC from baseline up to month 12 of $\geqslant 0.10 \mathrm{~L}$; a parameter that was expected to have a normal distribution with a standard deviation of $0.20 \mathrm{~L}$.

\section{Methods}

Within 4 weeks of screening and using a centralised system (Interactive Voice/Web Response System; S-Clinica, Brussels, Belgium), subjects were randomised 2:1 to oral macitentan (10 $\mathrm{mg}$ once daily in the morning) or placebo. The first intake of study medication took place at the study centre, at the time of randomisation and after successful completion of all assessments. Subjects could receive study medication in addition to usual background therapy for IPF (if present and where permitted by the protocol); background therapy was to remain at a constant dose throughout the investigational period.

Both tablets and packaging for macitentan and placebo were indistinguishable. Investigators, study staff, subjects, monitors and the sponsor remained blinded to treatment assignment throughout the trial. Only after study closure were randomisation codes made available for data analysis.

\section{Analysis}

Outcome measures

The primary efficacy end-point was the change in FVC from baseline up to month 12 for macitentan versus placebo. Superiority of macitentan was defined as a placebo-corrected mean $\pm \mathrm{SD}$ difference of $\geqslant 0.10 \pm 0.20 \mathrm{~L}$.

The secondary efficacy end-point was the time to first occurrence of IPF worsening or death (all causes) up to the end of study. IPF worsening was defined as 1) worsening of pulmonary function test (PFT) results (i.e. showing a decrease from baseline in absolute $\mathrm{FVC} \geqslant 10 \%$ and a decrease from baseline in absolute corrected $D$ LCO $\geqslant 15 \%$ and which were not refuted by a confirmatory test after $\geqslant 4$ weeks) or 2) acute respiratory decompensation of IPF (i.e. an unexplained rapid deterioration of a subject's condition over a period of $<4$ weeks with an increasing shortness of breath requiring oxygen supplementation of $\geqslant 5 \mathrm{~L} \cdot \mathrm{min}^{-1}$ to maintain a resting oxygen saturation $\geqslant 90 \%$ or arterial oxygen pressure $\geqslant 55 \mathrm{mmHg}$ (at sea level) or $50 \mathrm{mmHg}$ (at high altitude)). Subjects who were unable to perform PFTs due to worsened IPF were considered to have worsening results unless this was disproved by a subsequent assessment.

Exploratory efficacy end-points included: 1) the evaluation of TDI [13] up to month 12 and the betweengroup difference in the numbers of subjects with TDI $\geqslant 1$ up to month $12 ; 2$ ) the change from baseline up to month 12 in PFT results (specifically FEV1 and corrected DLCO); and 3) the change from baseline up to month 12 in composite physiologic index [14]. The placebo-corrected change in FVC from baseline up to month 12 in subpopulations of subjects grouped by baseline demographics and clinical characteristics (specifically age, sex, location, FVC at baseline and use of oral IPF therapies at baseline) was also explored. All exploratory end-points were defined a priori and before unblinding.

\section{Statistical methods}

Analysis of the primary efficacy end-point was performed on the "all-randomised set", which consisted of all randomised subjects regardless of whether or not they received study medication. The change in FVC from baseline up to month 12 was evaluated and the treatment comparison performed using the Wilcoxon rank-sum test with asymptotic approximation to a t-distribution. The null hypothesis was that there was no difference between interventions in the change from baseline up to month 12 in FVC, using a two-sided 0.05 type-I error level. No interim analyses of efficacy were conducted and there were no significant changes to the study following its commencement. The primary efficacy analysis was repeated on a "per-protocol set”, which included all randomised subjects who were without major protocol deviations.

For the primary end-point analysis, in subjects for whom values from assessment of FVC were missing, the following replacement rules were applied: 1) the last available post-baseline measurement obtained before 
month 12 was carried forward as a substitute for the missing value; 2) if a subject died before their assessment, a value of $0.0 \mathrm{~L}$ was substituted for the missing values; 3 ) for subjects who were alive and experienced disease worsening before month 12 but had no FVC assessment between the occurrence of the event and month 12, the worst value for the FVC (defined as the greatest percentage decrease observed between baseline and the considered time point for all subjects in the same analysis set) was imputed for the missing value; 4) for subjects who were alive and experienced no disease worsening before month 12 but for whom no post-baseline value was available, the mean percentage change at month 12 of observed values (including carry-forward values) in the analysis set was applied to the baseline FVC value. An exploratory repeated measurement analysis (random coefficient model) to investigate the changes in FVC from baseline over the main study period was performed.

The secondary efficacy end-point was evaluated in the all-randomised set. Data were summarised by means of Kaplan-Meier estimates of the proportion of subjects without the event at different time points displayed with $95 \%$ confidence limits (CL). Log-rank testing and derivation of a hazard ratio up to the end of the study were planned. Due to unmet assumptions on proportionality of hazards of treatments, post hoc treatment effect estimates by log rank and hazard ratio up to month 12 were determined (censoring was applied before the treatment mortality curves crossed).

Exploratory end-points were evaluated in the all-randomised set. Dichotomous variables were expressed as incidence and relative risk of the macitentan group towards placebo with 95\% CL. Continuous variables were expressed using summary statistics (mean, median, standard deviation, range and 95\% CL).

Safety analyses were performed on the all-randomised set, with data being summarised descriptively.

TABLE 1 Baseline demographics and clinical characteristics

\begin{tabular}{|c|c|c|}
\hline & Macitentan $10 \mathrm{mg}$ & Placebo \\
\hline Subjects $n$ & 119 & 59 \\
\hline Male & $84(70.6)$ & $37(62.7)$ \\
\hline \multirow[t]{2}{*}{ Age years } & $66(37-84)$ & $64(49-81)$ \\
\hline & $65.1 \pm 7.85$ & $64.5 \pm 6.32$ \\
\hline \multicolumn{3}{|l|}{ IPF medical status } \\
\hline Duration of symptoms days & $786(151-4348)$ & $771(60-3180)$ \\
\hline Duration since SLB-confirmed diagnosis days & $213(3-1870)$ & $114(2-1440)$ \\
\hline Digital clubbing & $23(19.3)$ & $8(13.6)$ \\
\hline \multicolumn{3}{|l|}{ Smoking } \\
\hline Never-smoker & 47 (39.5) & 22 (37.3) \\
\hline Ex-smoker & 67 (56.3) & $37(62.7)$ \\
\hline Current smoker & $5(4.2)$ & $0(0.0)$ \\
\hline \multicolumn{3}{|l|}{ Location } \\
\hline USA & $37(31.1)$ & 18 (30.5) \\
\hline Non-USA & $82(68.9)$ & $41(69.5)$ \\
\hline \multirow[t]{2}{*}{ FVC L } & $2.83(1.24-5.42)$ & $2.74(1.36-6.61)$ \\
\hline & $2.88 \pm 0.83$ & $2.79 \pm 0.78$ \\
\hline \multirow[t]{2}{*}{ FVC \% pred } & $76.1(47.7-121.1)$ & $73.8(54.0-126.4)$ \\
\hline & $76.5 \pm 15.6$ & $74.8 \pm 14.6$ \\
\hline \multirow[t]{2}{*}{ Corrected DLCo $\mathrm{mmol} \cdot \mathrm{kPa}^{-1} \cdot \mathrm{min}^{-1}$} & $4.16(2 . \overline{3} 7-8.75)$ & $4.13(2 . \overline{2} 5-8.70)$ \\
\hline & $4.55 \pm 1.49$ & $4.35 \pm 1.32$ \\
\hline \multirow[t]{2}{*}{ Corrected DLCo \% pred } & $46.5(26.3 \text { to } 90.7)^{\#}$ & $43.7(30.7-76.1)$ \\
\hline & $47.8 \pm 13.4^{\#}$ & $45.6 \pm 11.2$ \\
\hline Oral corticosteroid use & $28(23.5)$ & $15(25.4)$ \\
\hline Daily dose mg prednisone equivalent & $10.4 \pm 3.42$ & $12.1 \pm 5.17$ \\
\hline $\mathrm{N}$-acetylcysteine use & $19(16.0)$ & $13(22.0)$ \\
\hline Daily dose mg & $600 \pm 0.0$ & $592 \pm 27.7$ \\
\hline \multicolumn{3}{|l|}{ Blood oxygenation $\mathrm{mmHg}$} \\
\hline $\mathrm{PaO}_{2}$ & $77.9 \pm 11.5^{\bullet}$ & $80.2 \pm 15.0$ \\
\hline $\mathrm{PA}-\mathrm{aO}_{2}$ & $21.2 \pm 10.8^{+}$ & $19.6 \pm 11.3^{\S}$ \\
\hline
\end{tabular}

Data are presented as $\mathrm{n}(\%)$, median (range) or mean $\pm \mathrm{SD}$, unless otherwise stated. IPF: idiopathic pulmonary fibrosis; SLB: surgical lung biopsy; FCV: forced vital capacity; DLCO: diffusing capacity of the lung for carbon monoxide; \% pred: \% predicted; $\mathrm{PaO}_{2}$ : arterial oxygen tension; $\mathrm{PA}_{-} \mathrm{aO}_{2}$ : alveolar-arterial oxygen tension difference. ${ }^{\#}: n=115 ;{ }^{\bullet}: n=113 ;{ }^{+}: n=107 ;^{\S}: n=55$. 


\section{Results}

\section{Subject population}

The trial started on May 19, 2009. A total of 178 subjects were enrolled from 48 centres in 12 countries (Australia $(n=27)$, Canada $(n=15)$, France $(n=26)$, Germany $(n=13)$, Israel $(n=7)$, Italy $(n=8)$, Slovenia $(n=2)$, South Africa $(n=2)$, Spain $(n=11)$, Sweden $(n=1)$, Turkey $(n=11)$ and USA $(n=55))$. Of the 178 randomised subjects, 119 were allocated to macitentan and 59 to placebo. Baseline demographics and clinical characteristics were well balanced between treatment groups (table 1). Disposition of the study subjects is shown in figure 1. In accordance with the protocol, end of study was declared after the last randomised subject had received 12 months of treatment. The trial was completed on June 30, 2011.

27 macitentan-treated subjects and 10 placebo recipients were excluded from the per-protocol set. In each arm of the trial the principal reason for exclusion from the per-protocol set was a change in concomitant medications for IPF without documented worsening of the underlying disease.

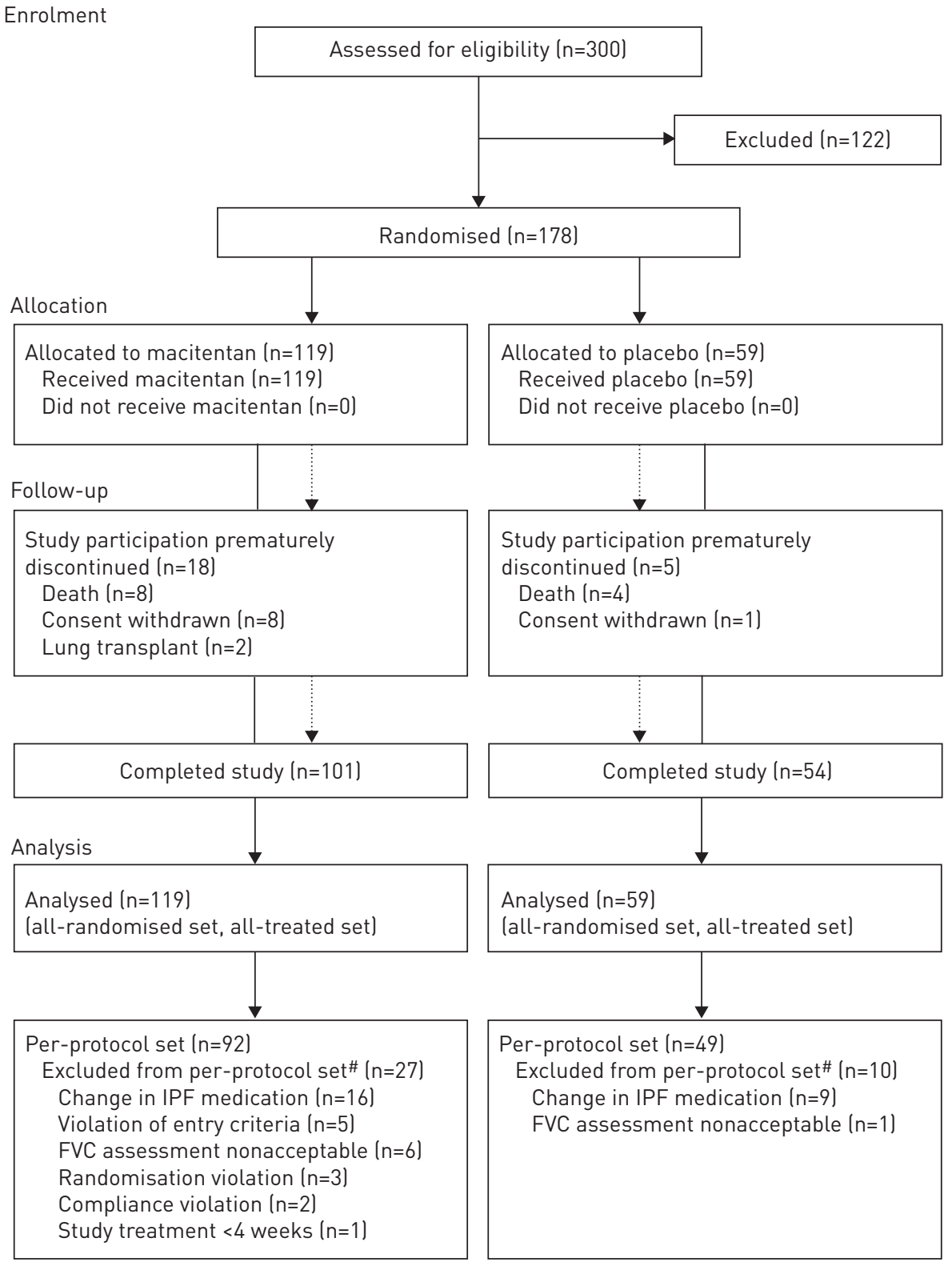

FIGURE 1 Disposition of study subjects. IPF: idiopathic pulmonary fibrosis; FVC: forced vital capacity. " : subjects may have exhibited more than one reason for exclusion. 


\section{Efficacy measurements}

Primary end-point: change from baseline up to month 12 in FVC

The median $(95 \% \mathrm{CL})$ change from baseline up to month 12 in absolute FVC was $-0.20 \mathrm{~L}(-0.29--0.16 \mathrm{~L})$ in the macitentan arm and $-0.20 \mathrm{~L}(-0.28--0.13 \mathrm{~L})$ in the placebo arm. The median $(95 \% \mathrm{CL})$ treatment effect of macitentan on FVC was $0.00 \mathrm{~L}(-0.09-0.08 \mathrm{~L})$. The mean change from baseline up to month 12 in absolute FVC, the mean treatment effect of macitentan on FVC and summary statistics pertaining to these estimates are shown in online supplementary table S1).

When analysis of the primary end-point was repeated in the per-protocol set, results were consistent with those of the all-randomised set (data not shown).

\section{Time to first occurrence of IPF worsening or death}

In total, 35 macitentan-treated subjects $(29.4 \%)$ and 17 placebo recipients (28.8\%) experienced at least one occurrence of IPF worsening or death. An early trend favouring placebo was observed for time to IPF worsening up to month 12 (post hoc HR 1.56, 95\% CL 0.73-3.33; log-rank p=0.2428) (fig. 2). This difference was mainly driven by a numerically greater number of subjects in the macitentan arm who exhibited early acute respiratory decompensation of IPF (seven (5.9\%) subjects) versus placebo (one (1.7\%) subject).

Overall, in both treatment groups, the majority of events were recorded as IPF worsening with a deterioration in PFT results (macitentan $n=25$ (21.0\%); placebo $n=14(23.7 \%)$ ). Death, when considered for the analysis of the secondary end-point only, occurred in three $(2.5 \%)$ subjects in the macitentan group and two $(3.4 \%)$ subjects in the placebo group.

Kaplan-Meier estimates of the time to first occurrence of IPF worsening or death at months 4, 8, 12, 20 and 24 are shown in online supplementary table S2. Results of the sensitivity analysis (random coefficient model) conducted post hoc also showed no treatment effect of macitentan on the change in FVC over time (data not shown). Data showing the causes of first occurrences of disease worsening and death up to month 12 and up to end of study are shown in online supplementary table S3.

\section{Pulmonary function tests}

The median changes from baseline up to month 12 in absolute FVC, absolute FEV1 and corrected DLCO are shown in table 2. The median treatment difference between macitentan and placebo for FEV1 was small and did not reach statistical significance $(-0.03$ L, 95\% CL $-0.10-0.04 ; \mathrm{p}=0.45)$. Similarly, for corrected DLCO the median treatment difference between macitentan and placebo was small and not statistically significant (0.03 $\mathrm{mmol} \cdot \mathrm{kPa}^{-1} \cdot \mathrm{min}^{-1}, 95 \%$ CL $\left.-0.17-0.23 ; \mathrm{p}=0.76\right)$.

The median change from baseline up to month 12 in the composite physiologic index is shown in table 3. The median treatment difference between macitentan and placebo for the composite physiologic index was small and did not reach statistical significance $(-0.4,95 \%$ CL $-2.4-1.6 ; \mathrm{p}=0.66)$.

FIGURE 2 Kaplan-Meier estimate for time to first event of idiopathic pulmonary fibrosis worsening or death. Data are Kaplan-Meier estimates with $95 \%$ confidence limits at 4 month intervals from subjects in the allrandomised set.

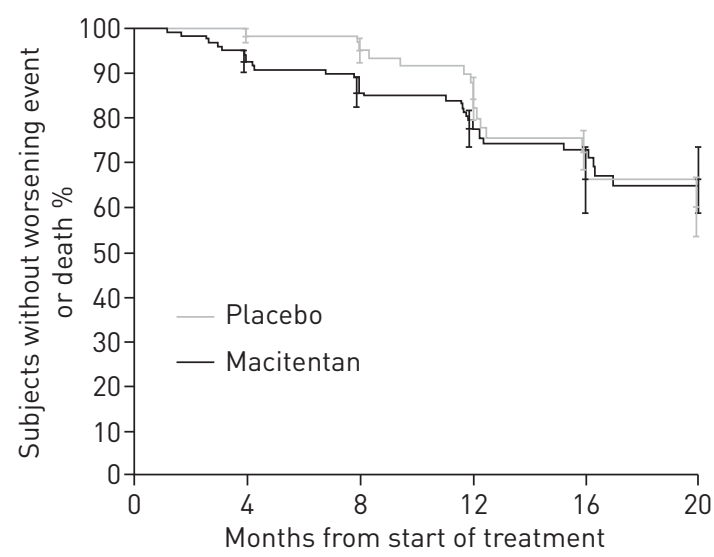

Placebo $n$

At risk 59

With event 0

Macitentan $n$

At risk 119

With event 0

112

$\begin{array}{ccccc}59 & 57 & 44 & 22 & 8 \\ 1 & 3 & 9 & 16 & 16 \\ 112 & 103 & 81 & 43 & 14 \\ 9 & 17 & 26 & 30 & 35\end{array}$


TABLE 2 Change from baseline up to month 12 in median absolute forced vital capacity (FVC), absolute forced expiratory volume in $1 \mathrm{~s}(\mathrm{FEV} 1)$ and corrected diffusing capacity of the lung for carbon monoxide (DLCo)

\begin{tabular}{|c|c|c|}
\hline & Macitentan $10 \mathrm{mg}$ & Placebo \\
\hline Subjects $n$ & 119 & 59 \\
\hline \multicolumn{3}{|l|}{ FVC L } \\
\hline Baseline & $2.83(1.24-5.42)$ & $2.74(1.36-4.61)$ \\
\hline Up to month 12 & $2.57(0.00-5.16)$ & $2.40(0.00-4.67)$ \\
\hline Change from baseline & $-0.20(-2.86-0.42)$ & $-0.20(-4.12-0.62)$ \\
\hline \multicolumn{3}{|l|}{ FEV ${ }_{1}^{\# L}$} \\
\hline Baseline & $2.34(0.93-4.24)$ & $2.23(1.12-3.84)$ \\
\hline Up to month 12 & $2.14(0.00-4.34)$ & $1.96(0.00-3.85)$ \\
\hline Change from baseline & $-0.18(-2.47-0.35)$ & $-0.15(-3.46-0.51)$ \\
\hline \multicolumn{3}{|c|}{ Corrected $D \mathrm{LCO}^{\#} \mathrm{mmol} \cdot \mathrm{KPa}^{-1} \cdot \mathrm{min}^{-1}$} \\
\hline Baseline & $4.18(2.38-8.75)$ & $4.13(2.25-8.70)$ \\
\hline Up to month 12 & $3.82(0.00-8.46)$ & $3.54(0.00-7.27)$ \\
\hline Change from baseline & $-0.40(-6.64-1.07)$ & $-0.42(-5.17-0.90)$ \\
\hline
\end{tabular}

Data are presented as median (range), unless otherwise stated. For the FVC analysis, eight (6.8\%) subjects in the macitentan group had no post-baseline data: for four (3.4\%) subjects the worst values were imputed and for four $(3.4 \%)$ subjects the mean percentage change was imputed. In subjects with post-baseline data, five $(4.2 \%)$ macitentan-treated subjects and four $(6.8 \%)$ placebo recipients had data carried forward and three (2.5\%) macitentan-treated subjects and two (3.4\%) placebo recipients had worst values substituted for missing data. For the FEV1 analysis, six $(5.2 \%)$ subjects in the macitentan group had no post-baseline data and the worst values were imputed. In subjects with post-baseline data, three $(2.6 \%)$ macitentan-treated subjects and four $(6.8 \%)$ placebo recipients had data carried forward and three (2.6\%) macitentan-treated subjects and two (3.4\%) placebo recipients had worst values substituted for missing data. For the DLCo analysis, four (3.5\%) subjects in the macitentan group had no post-baseline data and the worst value was imputed. In subjects with post-baseline data, six (5.2\%) macitentan-treated subjects and four (6.8\%) placebo recipients had data carried forward and four (3.5\%) macitentan-treated subjects and three $(5.1 \%)$ placebo recipients had worst values substituted for missing data. ${ }^{\#}: \mathrm{n}=115$ in macitentan treatment arm.

Data showing mean changes from baseline up to month 12 and mean treatment differences between macitentan and placebo in absolute FEV1 and corrected DLCO are shown in online supplementary table S4.

\section{Transition dyspnoea index}

The median (95\% CL) TDI at month 12 was - $1.0(-3.0-0.0)$ for macitentan-treated subjects and $0.0(-2.0-$ $0.0)$ for placebo recipients. The number of subjects with TDI $\geqslant 1$ in the macitentan arm was $27(23.5 \%)$ and in the placebo arm was 17 (28.8\%); a relative risk reduction of 0.19 (95\% CL -0.37-0.52; p=0.47).

\section{Efficacy in subject subgroups}

Results of exploratory analyses of placebo-corrected changes from baseline up to month 12 in FVC among subpopulations of subjects grouped by age, sex, location, FVC at baseline and use of oral IPF therapies at baseline were similar to those observed in the primary analysis (fig. 3 ).

\section{TABLE 3 Change from baseline up to month 12 in composite physiologic index (CPI)}

\begin{tabular}{lcc} 
& Macitentan $\mathbf{1 0} \mathbf{~ m g}$ & Placebo \\
\hline Subjects $\mathbf{n}$ & $115^{\#}$ & $58^{\#}$ \\
CPI & & $48.3(17.4-70.5)$ \\
$\quad$ Baseline & $46.5(1.3-73.7)$ & $55.1(17.5-80.9)$ \\
$\quad$ Up to month 12 & $50.7(-9.2-80.9)$ & $4.2(-6.7-41.1)$ \\
$\quad$ Change from baseline & $3.7(-16.7-49.5)$ &
\end{tabular}

Data are presented as median (range), unless otherwise stated. ${ }^{\#}$ : data from subjects in the all-treated set who had a valid baseline measure of CPI. 


\begin{tabular}{|c|c|c|c|c|c|c|c|c|c|c|c|c|c|}
\hline & Macitentan & & Median & $95 \% \mathrm{CL}$ & & Fav & ars pl & cebo & & Favo & $\mathrm{ma}$ & ntan & \\
\hline & $\mathrm{n}$ & $\mathrm{n}$ & L & & -0.4 & -0.3 & -0.2 & -0.1 & 0.0 & 0.1 & 0.2 & 0.3 & 0.4 \\
\hline All subjects & 119 & 59 & 0.00 & $-0.09-0.08$ & & & & $\mapsto$ & & & & & \\
\hline $\begin{array}{l}\text { Age years } \\
\leqslant \text { median (65) }\end{array}$ & 58 & 34 & 0.07 & $-0.05-0.18$ & & & & & & & & & \\
\hline$>$ median (65) & 61 & 25 & -0.08 & $-0.22-0.04$ & & & & 1 & & & & & \\
\hline Sex & & & & & & & & & & & & & \\
\hline Male & 84 & 37 & 0.03 & $-0.08-0.16$ & & & & $\vdash$ & & & & & \\
\hline Female & 35 & 22 & -0.04 & $-0.15-0.07$ & & & & & & & & & \\
\hline Location & & & & & & & & & & & & & \\
\hline USA & 37 & 18 & 0.05 & $-0.15-0.26$ & & & & & & & & & \\
\hline Non-USA & 82 & 41 & -0.02 & $-0.11-0.07$ & & & & $\longmapsto$ & & & & & \\
\hline FVC at baseline & & & & & & & & & & & & & \\
\hline$\leqslant$ median (2.8) & 57 & 32 & 0.02 & $-0.11-0.12$ & & & & $\vdash$ & - & $\longrightarrow$ & & & \\
\hline > median (2.8) & 62 & 27 & -0.01 & $-0.15-0.12$ & & & & & & & & & \\
\hline Oral IPF therapi & & & & & & & & & & & & & \\
\hline Yes & 37 & 19 & 0.14 & $-0.01-0.29$ & & & & & & & & -1 & \\
\hline No & 82 & 40 & -0.07 & $-0.17-0.04$ & & & & & & & & & \\
\hline & & & & & -0.4 & -0.3 & -0.2 & -0.1 & 0.0 & 0.1 & 0.2 & 0.3 & 0.4 \\
\hline
\end{tabular}

FIGURE 3 Placebo-corrected median changes from baseline up to month 12 in forced vital capacity (FVC) in different subpopulations of study subjects. Data are from subjects in the all-randomised set. IPF: idiopathic pulmonary fibrosis.

\section{Safety and tolerability}

The median (range) exposure to macitentan was $14.5(0.0-24.6)$ months and to placebo was 15.0 (6.3$24.3)$ months. A total of $91(76.5 \%)$ macitentan-treated subjects and $48(81.4 \%)$ placebo recipients were exposed to investigational treatments for $\geqslant 12$ months. From the beginning of treatment until up to 28 days after study drug discontinuation, 116 subjects in the macitentan group (97.5\%) and 58 subjects in the placebo group (98.3\%) experienced at least one adverse event. Adverse events occurring in $\geqslant 10 \%$ of macitentan-treated subjects are shown in table 4 . A total of $15(12.6 \%)$ macitentan-treated subjects and seven (11.9\%) placebo recipients experienced adverse events that led to the premature discontinuation of study treatment. $37(31.1 \%)$ macitentan-treated subjects and $20(33.9 \%)$ placebo recipients experienced at least one serious adverse event. The most common serious adverse events are shown in table 5. In total, $3.4 \%$ of macitentan-treated subjects and $5.1 \%$ of placebo recipients exhibited alanine and/or aspartate aminotransferase elevations over three times the upper limit of normal. Observations following other assessments of the tolerability of investigational treatments are available as online supplementary material.

In total, 13 subjects died during treatment or up to 28 days after the discontinuation of study drug: nine (7.6\%) subjects in the macitentan arm and four (6.8\%) in the placebo arm. The most common causes of death were IPF worsening (macitentan $\mathrm{n}=2(1.7 \%)$ and placebo $\mathrm{n}=4(6.8 \%)$ ) and respiratory failure (macitentan $\mathrm{n}=3(2.5 \%)$ and placebo $\mathrm{n}=1(1.7 \%))$.

\section{TABLE 4 Adverse events occurring in $\geqslant 10 \%$ of macitentan-treated subjects}

\begin{tabular}{lcc} 
& Macitentan $\mathbf{1 0} \mathbf{~ m g}$ & Placebo \\
\hline Subjects & 119 & 59 \\
Worsening of IPF & $25(21.0)$ & $15(25.4)$ \\
Dyspnoea & $24(20.2)$ & $9(15.3)$ \\
Cough & $22(18.5)$ & $22(35.6)$ \\
Upper respiratory tract infection & $20(16.8)$ & $12(20.3)$ \\
Bronchitis & $16(13.4)$ & $9(15.3)$ \\
Peripheral oedema & $14(11.8)$ & $4(6.8)$ \\
Anaemia & $13(10.9)$ & $0(0.0)$ \\
\hline
\end{tabular}

Data are presented as $\mathrm{n}$ or $\mathrm{n}(\%)$. Data are from subjects in the all-randomised set. IPF: idiopathic pulmonary fibrosis. 
TABLE 5 Serious adverse events occurring in more than one macitentan-treated subject

Macitentan $10 \mathrm{mg}$

\begin{tabular}{lcc}
\hline Subjects & 119 & 59 \\
IPF worsening & $10(8.4)$ & $6(10.2)$ \\
Pneumonia & $6(5.0)$ & $2(3.4)$ \\
Respiratory failure & $4(3.4)$ & $2(3.4)$ \\
Hypoxia & $3(2.5)$ & $2(3.4)$ \\
Acute respiratory failure & $2(1.7)$ & $1(1.7)$ \\
Thrombocytopenia & $2(1.7)$ & $0(0.0)$ \\
\hline
\end{tabular}

Data are presented as $\mathrm{n}$ or $\mathrm{n}(\%)$. Data are from subjects in the all-randomised set. IPF: idiopathic pulmonary fibrosis.

\section{Discussion}

The MUSIC trial was a prospective, randomised, double-blind, multicentre, parallel-group, placebocontrolled, phase II proof-of-concept study to investigate the efficacy and safety of the dual ERA, macitentan, among study subjects with histologically proven IPF. The primary objective of the MUSIC trial, to demonstrate that macitentan $10 \mathrm{mg}$ once daily positively affected FVC versus placebo, was not met: no significant difference between treatments could be discerned in the primary end-point of change from baseline up to month 12 in FVC. In addition, no differences were observed between treatment groups in any of the secondary measures including time to IPF worsening or death and in exploratory investigations of treatment-related changes in PFT results. Long-term exposure to macitentan $10 \mathrm{mg}$ once daily was well tolerated, with an incidence of elevated hepatic aminotransferases that was similar to placebo.

While two previous trials of the dual ERA bosentan in subjects with IPF did not meet their primary endpoints $[8,9]$ and one trial of the single ERA ambrisentan was terminated due to a lack of efficacy [10], some beneficial effects of bosentan treatment were observed in dyspnoea and health-related quality of life in a subset of subjects who underwent surgical lung biopsy for diagnosis of IPF [8, 15]. The MUSIC trial, which was initiated before the results of the BUILD-3 trial of bosentan [9] were available for analysis, provided an opportunity to investigate the efficacy and tolerability of the dual ERA, macitentan, in a study population of subjects with IPF causing a mild-to-moderate impairment in pulmonary function.

In this trial, treatment of subjects with the dual ERA macitentan was well tolerated. More than threequarters of the subjects in the macitentan treatment arm were exposed to the drug for $\geqslant 12$ months. The profile of adverse events observed in macitentan-treated subjects was similar to that seen in the placebo group, especially with respect to the low rates of alanine and/or aspartate aminotransferase elevations over three times the upper limit of normal. A greater proportion of subjects treated with macitentan versus placebo exhibited peripheral oedema and anaemia. Fluid retention and anaemia are known to be associated with ERAs as a class of agents in general, with increased risks of these events having been reported previously in subjects with pulmonary arterial hypertension as well as chronic heart failure [16-21]. There was no difference between the two treatment arms with regards to the incidence of death; rather, the observed difference in risk between the groups up to month 12 was mainly driven by acute exacerbation. The reason for this is not known. The observed lower rate of cough among macitentan-treated subjects versus placebo recipients is also noteworthy, although we have no explanation for this.

In the present trial, the change from baseline up to month 12 in FVC was selected as the primary end-point. This end-point was selected since FVC and other resting PFT results are used routinely in the management of IPF. Furthermore, longitudinal decreases in FVC have been shown to be significant prognostic indicators in IPF in several studies [22-25]. One exploratory study showed a possible prognostic value of decreases in FVC of $\geqslant 5 \%$ over 3 months [26]. Another group estimated the minimal clinically important difference in FVC over a 24 -week period to be between $2 \%$ and $6 \%$ [25]. The selection of a mean relative difference in FVC between treatment arms of $0.10 \mathrm{~L}$ and a standard deviation of $0.20 \mathrm{~L}$ were considered, based on such reports $[25,26]$, to approximate a minimal clinically significant difference. The direction and magnitude of longitudinal changes in FVC and corrected DLCO observed in the MUSIC trial readily mirror those observed in several other studies in subjects with IPF [8, 9, 27, 28]. Similarly, the median increase from baseline up to month 12 in composite physiologic index observed in the present study was of a similar order of magnitude to that reported elsewhere among placebo recipients over a similar study duration [29]. 
Endothelin-1 has been implicated and may contribute to the fibrogenic processes underlying IPF [7]. However, efficacy could not be demonstrated in several clinical trials that investigated single and dual ERAs in subjects with IPF [8-10], including the present trial. Since IPF is a fibroproliferative disease in which several pathogenic mechanisms are implicated, it is plausible that the key to successful treatment may involve a combination of therapies targeting multiple pathways [30].

We acknowledge that the MUSIC trial had certain limitations. FVC is a reliable and responsive measure of clinical status in IPF, in which a decline of $2-6 \%$ pertains to clinical importance [25]. However, FVC is not fully validated as a surrogate measure for more meaningful outcomes, including survival [31]. As a result, the choice of FVC as the primary end-point in this phase II trial may be considered to be disadvantageous by some, in comparison with all-cause, nonelective hospitalisation or all-cause mortality [31]. The potential for variability within assessments of PFTs may also represent a limitation in the choice of a physiological end-point. In addition, due to unmet assumptions on proportionality of hazards of treatments, treatment effect estimates in the time-to-event analysis were determined post hoc. Furthermore, the lack of a dosefinding study meant that we were unable to ascertain the potential effects, if any, of higher doses of macitentan among the subjects who participated in the trial.

In conclusion, the primary objective of the MUSIC trial, to determine if macitentan positively affected the change in FVC from baseline up to month 12 versus placebo, was not met. No differences between treatment groups were observed in any of the secondary or exploratory end-points. Long-term exposure to macitentan was well tolerated with a similar, low incidence of elevated hepatic aminotransferases in each treatment group.

\section{Acknowledgements}

The authors express their gratitude to all investigators who participated in the MUSIC trial: N. Goh (Dept of Allergy, Immunology and Respiratory Medicine, The Alfred Hospital, Melbourne), A. Glanville (Heart and Lung Transplant Unit, St Vincent's Hospital, Darlinghurst), M. Musk (Advanced Lung Disease Unit, Royal Perth Hospital, Perth) and P. Hopkins (Thoracic Department, Prince Charles Hospital Lung Transplant, Chermside), Australia; D.C. Lien (University of Alberta Health Science Centre, Edmonton), C. Chan (University Health Network, Toronto), J.D. Rolf (Kelowna General Hospital, Respiratory and Allergy Cinic, Kelowna), P. Wilcox (St Paul's Hospital, Vancouver), P.G. Cox (St Joseph's Healthcare, Firestone Institute for Respiratory Health, Hamilton) and H. Manganas (Hopital Notre-Dame du Centre, Hospitalier de l'Universite de Montreal, Montreal), Canada; V. Cottin (Hôpital Cardiologique et Pneumologique Louis Pradel, Service de Pneumologie, Bron), D. Valeyre (Hopital Avicenne, Service de Pneumologie, Bobigny) and B. Walleart (Clinique des Maladies Respiratoires, CHRU Hopital Calmette, Lille), France; S. Andreas (Fachklinik für Lungenerkrankungen, Immenhausen), C. Neurohr (Klinikum der Universität München, Medizinische Klinik und Poliklinik 1, Großhadern Schwerpunkt Pneumologie, Munich), A. Guenther (Justus-Liebig-Universität Gießen, Zentrum für Innere Medizin, Medizinische Klinik II, Giessen), N. Schönfeld (Helios Klinikum Emil von Behring, Zentrum für Pneumologie und Thoraxchirurgie, Berlin) and A. Koch (Universitätsklinikum Köln, Herzzentrum/Klinik III für Innere Medizin (Kardiologie, Pneumologie, Angiologie und Intensivmedizin), Cologne), Germany; M. Kramer (Rabin Medical Centre, Pulmonary Institute, Petach Tikvah), R. Breuer (Institute of Pulmonology, Hadassah Medical Centre Ein Karem, Jerusalem), I. Ben-Dov (The Chaim Sheba Medical Center/The Institute of Pulmonology, Physiology and Exercise, Tel-Hashomer), G. Fink (Kaplan Medical Centre/Pulmonary Institute and Dept of Medicine, Rehovot) and Y. Schwarz (Tel Aviv Sourasky Medical Center, Dept of Pulmonary and Allergic Diseases, Tel-Aviv), Israel; C. Albera (Azienda Sanitaria Ospedaliera, San Luigi Gonzaga - Clinica Malattie Apparato Respiratorio, Turin), M. Confalonieri (Dipartimento di Pneumologia, Ospedale di Cattinara, Trieste), C. Saltini (Dipartimento di Medicina, Unità Operativa Complessa di Malattie dell'Apparato Respiratorio, Azienda Ospedaliera Universitaria Policlinico Tor Vergata di Roma, Rome) and S. Harari (U.O. Pneumologia, Ospedale San Giuseppe, Milan), Italy; M. Flezar, Bolnisnica Golnik, Golnik, Slovenia; M. Greenblatt and G.J. Ras, Centre for Chest Disease, Milpark Hospital, Johannesburg, South Africa; F. Morell (Neumologia, Hospital Universitatario Vall d'Hebron, Barcelona), J.L. Alvarez-Sala (Pneumology, Hospital Universitario Clinico San Carlos, Madrid), A. Xaubet (Pneumology Department, Hospital Clínic Barcelona, Barcelona), A. Sueiro (Hospital Universitario Ramón y Cajal, Madrid) and M.J. Linares (Pneumology, Fundacion Hospital Alcorcon, Alcorcon), Spain; M. Sköld, Lung Allergi forskningen, Karolinska Universitetssjukhuset Solna, Stockholm, Sweden; O. Kayacan (Dept of Chest Disease, Ankara University School of Medicine, Ankara) and N. Mogulkoc (Dept of Chest Disease, Ege University School of Medicine, Bornova-Izmir), Turkey; A. Chan (University of California Davis, UC Davis Medical Center, Main Hospital, Sacramento, CA), J. Chapman and J. Parambil (Cleveland Clinic Foundation, Cleveland, $\mathrm{OH}$ ), N. Ettinger (St Luke's Medical Group, Cardio-Pulmonary Associates, Chesterfield, MO), J. Golden (University of California - San Francisco, Ambulatory Care Center, San Francisco, CA), K.C. Meyer (University of Wisconsin, Hospital and Clinics, Madison, WI), J.J. Swigris (National Jewish Health, Denver, CO), G.L. Yung (University of California - San Diego, Medical Center, San Diego, CA), D. Antin-Ozerkis (Yale University School of Medicine, Winchester Chest Clinic, New Haven, CT), P.K. Mohabir (Stanford University Medical Center - Chest Clinics, Stanford, CA), L.J. Wesselius (Mayo Clinic Arizona, Scottsdale, AZ), J. de Andrade (Pulmonary, Allergy and Critical Care Medicine, University of Alabama at Birmingham, Birmingham, AL), F. Cordova (Temple Lung Center, Philadelphia, PA), Z. Safdar (Baylor College of Medicine, Houston, TX) and M. Wencel (Wichita Clinic, Wichita, KS), USA. The authors thank the members of the independent Data Safety Monitoring Board: H.J. Eisen (Drexel University College of Medicine, Philadelphia, PA, USA), C.M. O'Connor (Duke University Medical Center, Durham, NC, USA), B. Nashan (University Medical Centre Eppendorf, Hamburg, Germany) and D.L. DeMets (DL DeMets Consulting, Madison, WI, USA). The authors thank all contributors in the three central analysis laboratories and A. Gray (Elements Communications Ltd, Westerham, UK) for medical writing assistance, supported by Actelion Pharmaceuticals Ltd (Allschwil, Switzerland). 


\section{References}

1 Raghu G, Collard HR, Egan JJ, et al. An official ATS/ERS/JRS/ALAT statement: idiopathic pulmonary fibrosis: evidence-based guidelines for diagnosis and management. Am J Respir Crit Care Med 2011; 183: 788-824.

2 Nalysnyk L, Cid-Ruzafa J, Rotella P, et al. Incidence and prevalence of idiopathic pulmonary fibrosis: review of the literature. Eur Respir Rev 2012; 21: 355-361.

3 Demedts M, Behr J, Buhl R, et al. High-dose acetylcysteine in idiopathic pulmonary fibrosis. N Engl J Med 2005; 353: 2229-2242.

4 Taniguchi H, Ebina M, Kondoh Y, et al. Pirfenidone in idiopathic pulmonary fibrosis. Eur Respir J 2010; 35 : 821-829.

5 Noble PW, Albera C, Bradford WZ, et al. Pirfenidone in patients with idiopathic pulmonary fibrosis (CAPACITY): two randomised trials. Lancet 2011; 377: 1760-1769.

6 Richeldi L, Costabel U, Selman M, et al. Efficacy of a tyrosine kinase inhibitor in idiopathic pulmonary fibrosis. N Engl J Med 2011; 365: 1079-1087.

7 Fonseca C, Abraham D, Renzoni EA. Endothelin in pulmonary fibrosis. Am J Respir Cell Mol Biol 2011; 44: 1-10.

8 King TE Jr, Behr J, Brown KK, et al. BUILD-1: a randomized placebo-controlled trial of bosentan in idiopathic pulmonary fibrosis. Am J Respir Crit Care Med 2008; 177: 75-81.

9 King TE Jr, Brown KK, Raghu G, et al. BUILD-3: a randomized, controlled trial of bosentan in idiopathic pulmonary fibrosis. Am J Respir Crit Care Med 2011; 184: 92-99.

10 Raghu G, Behr J, Brown KK, et al. ARTEMIS-IPF: A placebo-controlled trial of ambrisentan in idiopathic pulmonary fibrosis. Am J Respir Crit Care Med 2012; 185: A3632.

11 Iglarz M, Landskroner K, Rey M, et al. Optimization of tissue targeting properties of macitentan, a new dual endothelin receptor antagonist, improves its efficacy in a rat model of pulmonary fibrosis associated with pulmonary arterial hypertension. Am J Respir Crit Care Med 2011; 183: A6445.

12 Idiopathic pulmonary fibrosis: diagnosis and treatment. International consensus statement. American Thoracic Society (ATS) and the European Respiratory Society (ERS). Am J Respir Crit Care Med 2000; 161: 646-664.

13 Mahler DA, Weinberg DH, Wells CK, et al. The measurement of dyspnea. Contents, interobserver agreement, and physiologic correlates of two new clinical indexes. Chest 1984; 85: 751-758.

14 Wells AU, Desai SR, Rubens MB, et al. Idiopathic pulmonary fibrosis: a composite physiologic index derived from disease extent observed by computed tomography. Am J Respir Crit Care Med 2003; 167: 962-969.

15 Raghu G, King TE Jr, Behr J, et al. Quality of life and dyspnoea in patients treated with bosentan for idiopathic pulmonary fibrosis (BUILD-1). Eur Respir J 2010; 35: 118-123.

16 Humbert M, Barst RJ, Robbins IM, et al. Combination of bosentan with epoprostenol in pulmonary arterial hypertension: BREATHE-2. Eur Respir J 2004; 24: 353-359.

17 Barst RJ, Langleben D, Frost A, et al. Sitaxsentan therapy for pulmonary arterial hypertension. Am J Respir Crit Care Med 2004; 169: 441-447.

18 Galié N, Badesch D, Oudiz R, et al. Ambrisentan therapy for pulmonary arterial hypertension. J Am Coll Cardiol 2005; 46: 529-535.

19 Vergouwen MD, Algra A, Rinkel GJ. Endothelin receptor antagonists for aneurysmal subarachnoid hemorrhage: a systematic review and meta-analysis update. Stroke 2012; 43: 2671-2676.

20 Badesch DB, Feldman J, Keogh A, et al. ARIES-3: ambrisentan therapy in a diverse population of patients with pulmonary hypertension. Cardiovasc Ther 2012; 30: 93-99.

21 Epstein BJ. Efficacy and safety of darusentan: a novel endothelin receptor antagonist. Ann Pharmacother 2008; 42: 1060-1069.

22 Collard HR, King TE Jr, Bartelson BB, et al. Changes in clinical and physiologic variables predict survival in idiopathic pulmonary fibrosis. Am J Respir Crit Care Med 2003; 168: 538-542.

23 Latsi PI, du Bois RM, Nicholson AG, et al. Fibrotic idiopathic interstitial pneumonia: the prognostic value of longitudinal functional trends. Am J Respir Crit Care Med 2003; 168: 531-537.

24 Flaherty KR, Mumford JA, Murray S, et al. Prognostic implications of physiologic and radiographic changes in idiopathic interstitial pneumonia. Am J Respir Crit Care Med 2003; 168: 543-548.

25 du Bois RM, Weycker D, Albera C, et al. Forced vital capacity in patients with idiopathic pulmonary fibrosis: test properties and minimal clinically important difference. Am J Respir Crit Care Med 2011; 184: 1382-1389.

26 Taniguchi $\mathrm{H}$, Kondoh $\mathrm{Y}$, Ebina M, et al. The clinical significance of $5 \%$ change in vital capacity in patients with idiopathic pulmonary fibrosis: extended analysis of the pirfenidone trial. Respir Res 2011; 12: 93.

27 Martinez FJ, Safrin S, Weycker D, et al. The clinical course of patients with idiopathic pulmonary fibrosis. Ann Intern Med 2005; 142: 963-967.

28 Raghu G, Brown KK, Costabel U, et al. Treatment of idiopathic pulmonary fibrosis with etanercept: an exploratory, placebo-controlled trial. Am J Respir Crit Care Med 2008; 178: 948-955.

29 Behr J, Demedts M, Buhl R, et al. Lung function in idiopathic pulmonary fibrosis - extended analyses of the IFIGENIA trial. Respir Res 2009; 10: 101.

30 Bouros D. Pirfenidone for idiopathic pulmonary fibrosis. Lancet 2011; 377: 1727-1729.

31 Raghu G, Collard HR, Anstrom KJ, et al. Idiopathic pulmonary fibrosis: clinically meaningful primary endpoints in phase 3 clinical trials. Am J Respir Crit Care Med 2012; 185: 1044-1048. 